\title{
A determination of electroweak parameters at HERA
}

\author{
Zhiqing Zhang* \\ Laboratoire de l'Accélérateur Linéaire, IN2P3/CNRS et Université de Paris-Sud XI \\ BP34, F-91898 Orsay, France \\ E-mail: zhangzq@lal.in2p3.fr
}

\begin{abstract}
Using the deep inelastic $e^{ \pm} p$ charged and neutral current scattering cross sections previously published, a combined electroweak and QCD analysis is performed to determine electroweak parameters accounting for their correlation with parton distributions. The data used have been collected by the H1 experiment in 1994-2000 and correspond to an integrated luminosity of $117.2 \mathrm{pb}^{-1}$. A first measurement at HERA is made of the light quark weak couplings to the $Z^{0}$ boson. An improved measurement is obtained of the $W$ propagator mass in charged current $e p$ scattering. The weak mixing angle $\sin ^{2} \theta_{W}$ is determined in the on-mass-shell renormalization scheme.
\end{abstract}

European Physical Society

HEP2005 International Europhysics Conference on High Energy Physics EPS (July 21st-27th 2005) in Lisboa, Portugal

\footnotetext{
${ }^{*}$ On behalf of the H1 Collaboration.
} 


\section{The experimental facts and analysis strategies}

In the first phase of HERA operation (HERA-I) with the unpolarized $e^{ \pm}$beam colliding with the proton beam, the $\mathrm{H} 1$ experiment has collected three major data samples of $e^{+} p$ in years from 1994 to 1997 at a center-of-mass energy of $301 \mathrm{GeV}, e^{-} p$ in 1998-1999 and $e^{+} p$ in 19992000 at $319 \mathrm{GeV}$. The corresponding integrated luminosities are $35.6 \mathrm{pb}^{-1}, 16.4 \mathrm{pb}^{-1}$ and 65.2 $\mathrm{pb}^{-1}$, respectively. These data have been used to measure neutral current (NC) and charged current (CC) cross sections covering more than 4 orders of magnitude in both $Q^{2}$, the negative four-momentum transfer squared, and Bjorken $x$. The large kinematic coverage and the different flavor sensitivity of the $e^{ \pm} p$ NC and CC cross section data have enabled 5 sets of parton distribution functions (PDF) to be determined simultaneously in a previous QCD analysis [1]. These five PDF sets are the gluon, up-type and down-type quarks and their anti-quarks distributions.

The inclusive NC and CC cross sections are not only sensitive to PDFs but also to electroweak (EW) parameters. Indeed, the NC cross sections at high $Q^{2}$ depend on up- and down-type quark couplings to the $Z^{0}$ boson, $a_{q}$ and $v_{q}(q=u, d)$, via structure functions, whereas the shape of the CC cross sections as a function of $Q^{2}$ is controlled by the propagator mass $\left(M_{\text {prop}}\right)$ of the $W$ boson. It is thus natural to extend the QCD analysis of [1] into a combined EWPDF analysis so that EW parameters can be determined together with the PDFs taking properly into account the small but non-negligible correlation between them.

This is precisely the strategy chosen in [2,3], namely using the same parameterization forms for the five PDF sets for the QCD part. The QCD analysis is performed using the DGLAP evolution equations [4] at next-to-leading order in the modified minimal subtraction renormalization scheme. All quarks are taken as massless. Several combined EW-PDF fits are performed either in a model independent way (fits $a_{u}-v_{u}-a_{d}-v_{d}-\mathrm{PDF}$ and $G-M_{\text {prop }}$-PDF) or within the Standard Model (SM, fits $M_{W}-\mathrm{PDF}$ and $m_{t}-\mathrm{PDF}$ ).

\section{First results on light quark couplings to the $Z^{0}$ boson at HERA}

The sensitivity on the quark couplings at HERA stems from the $\gamma Z$ interference and $Z^{0}$ exchange contributions in NC interactions at high $Q^{2}$. The results of the combined $a_{u}-V_{u}-a_{d^{-}}-V_{d^{-}}$ PDF fit are shown in Fig.1 and compared with similar results obtained recently by the CDF experiment [5] and combined LEP experiments [6]. The HERA determination has comparable precision to that from the Tevatron. These determinations are sensitive to $u$ and $d$ quarks separately, contrary to other measurements of the light quark- $Z^{0}$ couplings in $v N$ scattering [7] and atomic parity violation [8] on heavy nuclei. They also resolve any sign ambiguity and the ambiguities between $v_{q}$ and $a_{q}(q=u, d)$ of the determinations based on observables measured at the $Z^{0}$ resonance [6].

The HERA precision is expected to improve significantly with the data from HERA-II taken at higher luminosity. The longitudinally polarized $e^{ \pm}$beams at HERA-II will also provide additional sensitivity in constraining the vector couplings $v_{q}$. 

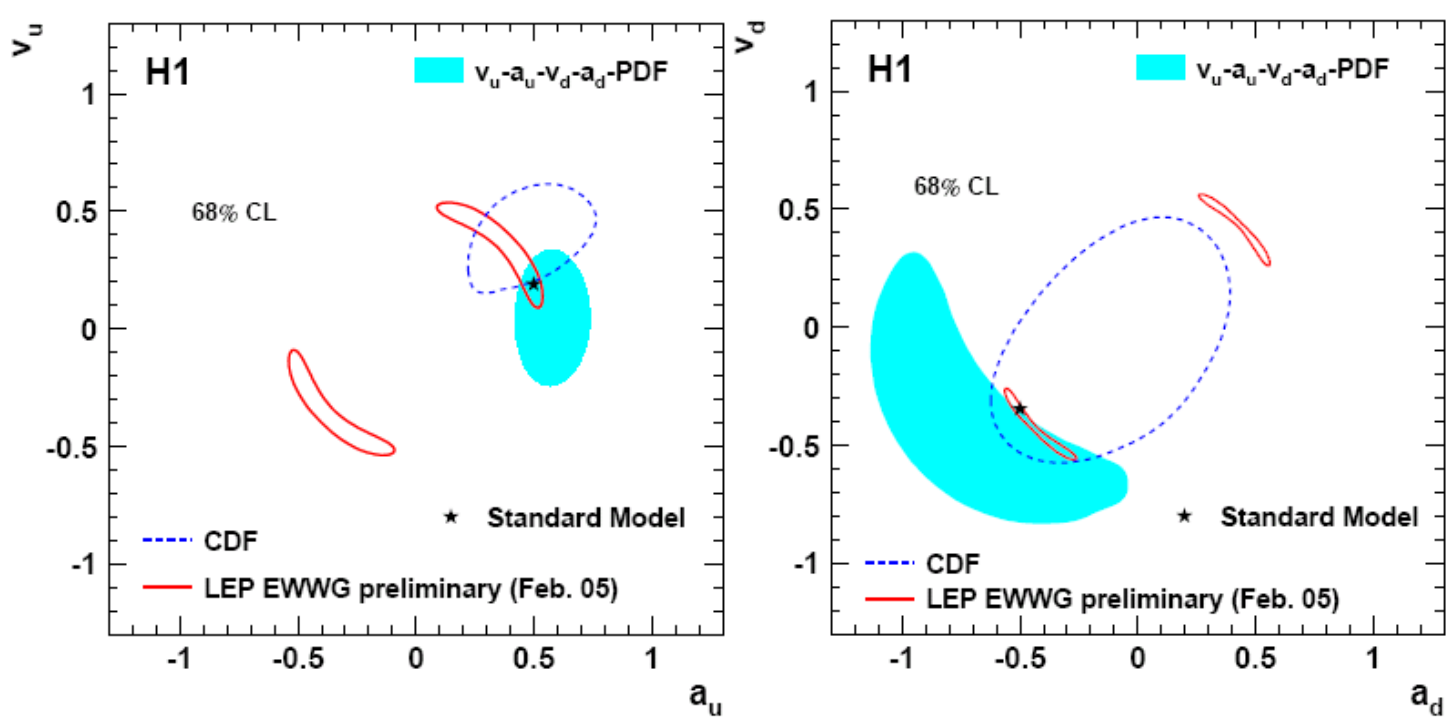

FIGURE 1. H1 results (shaded area) at $68 \%$ confidence level (CL) on the couplings of $u$ quark (left) and $d$ quark (right) to $Z^{0}$ in comparison with similar results from CDF (dashed curves) and LEP (full curves).

\section{Improved $W$ propagator mass measurement at HERA}

The cross section data allow a simultaneous determination of the Fermi coupling constant $G_{F}$ and the $W$ boson mass, and of the PDFs ( $G-M_{\text {prop }}$ PDF fit). When treating $G$ and $M_{\text {prop }}$ as independent parameters, the sensitivity on $G$ and $M_{\text {prop }}$ originates respectively from the normalization and $Q^{2}$ dependence of the CC cross sections. The result of the fit is shown in Fig.2 as the shaded area.

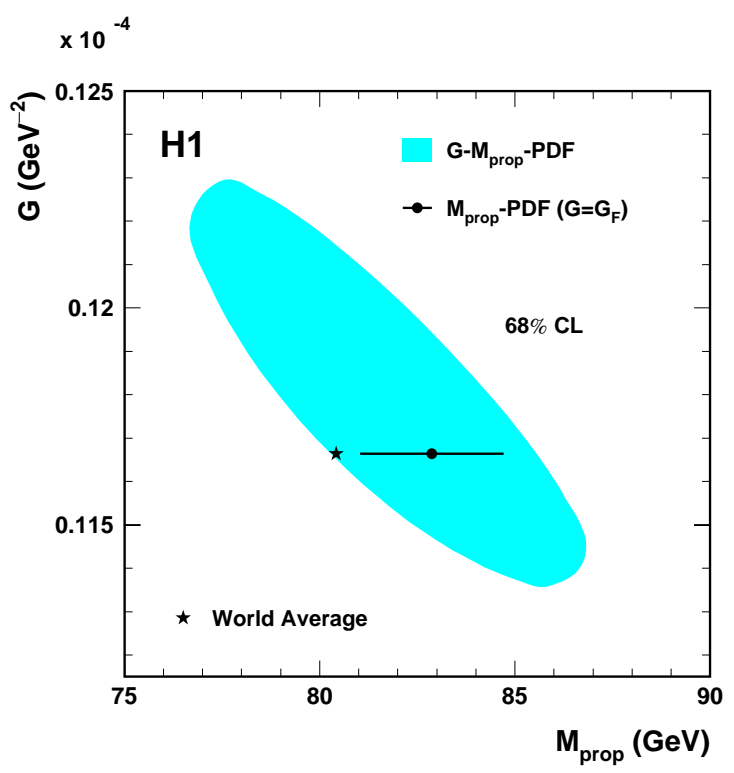

FIGURE 2. The result of the fit to $G$ and $M_{\text {prop }}$ at $68 \%$ confidence level (CL) shown as the shaded area. The world average values are indicated with the star symbol. Fixing $G$ to $G_{F}$, the fit results in a measurement of the propagator mass $M_{\text {prop }}$ shown as the circle with the horizontal error bar. 
Fixing $G$ to the measured $G_{F}$ value [9], one gets a determination of $M_{\text {prop }}$, also shown in Fig.1, $M_{\text {prop }}=82.87 \pm\left. 1.82_{\text {exp }-0.16}^{+0.30}\right|_{\text {model }} \mathrm{GeV}$ where the first error is experimental and the second corresponds to uncertainties due to input parameters and model assumptions as introduced in Table 5 in [1] (e.g., the variation of $\alpha_{s}=0.1185 \pm 0.0020$ ). This determination differs from all previous ones in the treatment of the correlation between $M_{\text {prop }}$ and PDFs and represents the most accurate measurement so far of the CC propagator mass at HERA.

Within the SM, the Fermi coupling constant $G_{F}$ is connected with the $W$ boson mass $M_{W}$ through a relation which contains EW radiative corrections including quadratic (logarithmic) dependence on the top quark mass $m_{t}$ (the Higgs mass $M_{H}$ ). A combined EW-PDF fit in the SM gives

$$
M_{W}=80.786 \pm\left. 0.205_{\text {exp }-0.029}^{+0.048}\right|_{\text {model }} \pm 0.025_{\delta m_{t}}-0.084_{\delta M_{H}} \pm 0.033_{\delta(\Delta r)} \mathrm{GeV}
$$

where the measured central value corresponds to using the world averaged values of $M_{Z}=$ $91.1876 \pm 0.0021 \mathrm{GeV}, m_{t}=178 \pm 4.3 \mathrm{GeV}$ and a Higgs mass of $120 \mathrm{GeV}$. The uncertainty on $M_{Z}$ has a negligible error on $M_{W}$ whereas the uncertainty on $m_{t}$ gives rise to the third quoted error on $M_{W}$. Varying $M_{H}$ from $120 \mathrm{GeV}$ to $300 \mathrm{GeV}$ results in the fourth error. The last error is due to higher order radiative correction uncertainties.

Together with the world average value of $M_{Z}$ given above, the result obtained on $M_{W}$ from Eqn.(1) represents an indirect determination of $\sin ^{2} \theta_{w}$ in the on-mass shell scheme: $\sin ^{2} \theta_{W}=0.2151 \pm 0.0040_{-0.0011}^{+0.0019}$ where the first error is experimental and the second is theoretical covering all remaining uncertainties in Eqn.(1). The uncertainty due to $\delta M_{Z}$ is negligible.

Fixing $M_{W}$ to the world average value and assuming $M_{H}=120 \mathrm{GeV}$, the fit $m_{t}-\mathrm{PDF}$ gives $m_{t}=108 \pm 44 \mathrm{GeV}$ where the uncertainty is experimental. The result represents the first determination of the top quark mass through loop effects in the ep data at HERA.

Again the precision of these determinations will be improved by a large amount as the best sensitivity comes from the CC $e^{-} p$ cross section which was measured from a very limited data sample at HEAR-I. Polarized $e^{-} p$ data corresponding to an increase of one order magnitude in the integrated luminosity from HERA-II are being taken.

\section{References}

[1] C. Adloff et al., [H1 Collaboration], Eur. Phys. J. C30 (2003) 1, [hep-ex/0304003].

[2] A. Aktas et al., [H1 Collaboration], accepted for publication in Phys. Lett. B., [hep-ex/0507080].

[3] B. Portheault, Ph.D. thesis (Mar. 2005), LAL 05-05 (IN2P3/CNRS, Université de Paris-Sud XI, Orsay), also available at http://wwwh1.desy.de/publications/theses list.html.

[4] G. Altarelli and G. Parisi, Nucl. Phys. B126 (1977) 298 and references therein.

[5] D. Acosta et al., [CDF Collaboration], Phys. Rev. D71 (2005) 052002 [hep-ex/0411059].

[6] http://lepewwg.web.cern.ch/LEPEWWG/plots/winter2005/.

[7] G.P. Zeller et al., [NuTeV Collaboration], Phys. Rev. Lett. 88 (2002) 091802, [hep-ex/0110059]; Erratum-ibid 90 (2003) 239902.

[8] S.C. Bennett and C.E. Wieman, Phys. Rev. Lett. 82 (1999) 2484, [hep-ex/9903022].

[9] Particle Data Group (S. Eidelman et al.), Phys. Lett. B592 (2004) 1. 